O IMPACTO DOS ÉSTERES DE FTALATO NO MEIO-AMBIENTE E NA SAÚDE HUMANA - SÃO ESSES COMPOSTOS, UM MAL NECESSÁRIO?

\title{
THE IMPACT OF PHTHALATE ESTERS IN THE ENVIRONMENTAL AND HUMAN HEALTH - ARE THESE COMPOUNDS, A NECESSARY EVIL?
}

\author{
DOS SANTOS, Marcel Silveira ${ }^{1}$ \\ Universidade Federal de Santa Catarina, Faculdade de Engenharia Química, Departamento de Engenharia \\ Química Ambiental, Campus Universitário-Córrego Grande, cep 88040-900, Florianópolis - SC, Brasil
}

(fone: +55 4837215230 )

e-mail: marcelsilveira_qmc@hotmail.com

Received 28 February 2011; received in revised form 08 August 2011; accepted 14 August 2011

\begin{abstract}
RESUMO
Ftalatos são um grupo de diésteres de ácido ftálico (dialquil ou alquil aril do ácido 1,2benzenodicarboxílico)-utilizados como plastificantes para conferir flexibilidade aos materiais poliméricos. Como não estão ligados quimicamente ao polímero, esses compostos podem migrar para o meio ambiente e, consequentemente, são encontrados nos alimentos, no ar, água, solo e no corpo humano. Esta mini-revisão descreve a problemática do uso desses compostos, sua toxicidade e impacto no meio ambiente. Apresenta um comentário sobre os quatro ftalatos mais debatidos atualmente, dados mensurados e legislação atual sobre esses compostos.
\end{abstract}

Palavras-chave: Éster ftálico; DEHP; Plastificante; Toxicidade; PVC.

\begin{abstract}
Phthalates are a group of diesters of phthalic acid (dialkyl or alkyl aryl esters of 1,2-benzenedicarboxylic acid) and they are primarily used as plasticizers (substances added to plastics to increase their flexibility). As the phthalates are not chemically bonded to the polymer, these compounds can migrate from the plastic material to the environment and, consequently, they are found in food, water, soil, air and in the human body. This article discusses the problem of using those compounds, their toxicity and impact on humans and environment. Comments about the four phthalates currently most discussed, measured values and current legislations are presented.
\end{abstract}

Keywords: Phthalic ester; DEHP; Plasticizer; Toxicity; PVC

PERIÓDICO TCHÊ QUÍMICA • www.periodico.tchequimica.com • Vol. 8 N. 16. - ISSN 1806-0374 (impresso) • ISSN 1806-9827 (CD-ROM) • ISSN 2179-0302 (meio eletrônico)

(C) 2011. Porto Alegre, RS. Brasil 


\section{INTRODUÇÃO}

A contaminação do meio ambiente tem sido apontada como um dos maiores problemas nos últimos anos. Como resultado da crescente conscientização desse problema, novas normas e legislações cada vez mais restritivas têm sido adotadas a fim de minimizar o impacto ambiental (Nogueira e Jardim, 1998).

A poluição nas diferentes fontes ambientais (água, solo e ar), que resulta como principal consequência do processo de industrialização ou simplesmente de atividades humanas, é um dos principais problemas que o homem tem para resolver ou, pelo menos, controlar. Os contaminantes ambientais podem ter diferentes características químicas e, em uma classificação preliminar, podem ser divididos em poluentes orgânicos, inorgânicos e espécies metálicas. Os contaminantes que possuem propriedades tóxicas ou cancerígenas têm recebido grande atenção atualmente (Kummrow, Umbuzeiro, 2006).

Um desses contaminantes é o famoso PVC - poli(cloreto de vinila), um material polimérico que se apresenta na sua forma original como um pó branco. Dentre os produtos à base de PVC, apenas $12 \%$ são considerados descartáveis com durabilidade de até 2 anos, $24 \%$ de 2 a 15 anos, e $64 \%$ entre 15 e 100 anos de vida útil (Instituto do PVC, 2010).

Quando contaminantes são tratados em sala de aula geralmente são abordados tópicos sobre emissões de $\mathrm{CO}_{2}$, liberação de metais pesados e esgoto em rios, utilização de agrotóxicos na agricultura e ainda poluição sonora e visual. Entretanto, o aumento da contaminação do meio ambiente pode estar "oculto" em muitos produtos de bens de consumo. Oculto, porque não são conhecidos da população em geral e nem por parte da maioria dos estudantes e, também, porque inicialmente alguns compostos eram considerados inofensivos e de extrema importância na manufatura de muitos produtos industrializados.

Um dos contaminantes que se encaixa nesse perfil são os ésteres ftálicos (alquil aril do ácido 1,2-benzenodicarboxílico). Os ftalatos são uma classe de substâncias químicas usados em uma ampla variedade de bens de consumo. Aproximadamente $80 \%$ de todos os ftalatos são utilizados como plastificantes (Gómez-Henz e
Aguilar-Caballos, 2003). Esses compostos são utilizados como plastificantes para modificar algumas propriedades dos plásticos, como flexibilidade, especialmente o PVC. Esses aditivos são encontrados em cosméticos e produtos de cuidados especiais como perfume, laquê, sabão, xampu, esmalte para unhas e hidratantes corporais; em plásticos flexíveis e brinquedos; capas de chuva, papéis de parede, cortinas plásticas, embalagens para alimentos. Ftalatos também são usados em acabamentos de madeira, detergentes, adesivos, tubulações plásticas, lubrificantes, utensílios médicos, solventes, inseticidas, materiais de construção e assoalhos (U.S. National Library of Medicine, 2009; Mackintosh et al., 2006).

A quantidade crescente das descobertas das aplicações deste aditivo em novos produtos fez que com a produção mundial de ftalatos aumentasse, do início da década de 90 até o ano de 2004, de 2 milhões para mais de 7 milhões de toneladas por ano, sendo um grande indicativo da sua importância econômica (Gómez-Henz e Aguilar-Caballos, 2003).

\section{TOXICIDADE}

A exposição humana aos ésteres de ftalato é significativa (U.S. National Library of Medicine, 2009). Esses compostos são encontrados em produtos alimentícios, na água, solo, ar e corpo humano pela migração a partir do material plástico para o ambiente ( $\mathrm{Ge}$ et al, 2007). A exposição humana a esses compostos se dá de diferentes formas: ingestão, inalação, absorção dérmica e até mesmo em injeções diretas como transfusões de sangue e aplicação de soro intradermicamente, devido à utilização de embalagens feitas de PVC (Chen et al, 2008).

Pessoas enfermas, especialmente crianças cujo organismo ainda está em desenvolvimento, podem ser particularmente sensíveis a esse tipo de exposição, uma vez que elas passam longo tempo no interior das casas em contato prolongado com superfícies que contêm ftalatos, seja por absorção dérmica e/ou inalação.

O contato pela pele pode ser a mais importante forma de absorção de ftalatos originários de produtos de cuidados pessoais, como o sabonete. (Pocurrull et al., 2001; Case Study, 2010). 


\section{IMPACTO SOBRE O AMBIENTE}

Ftalatos têm sido usados como aditivos em produtos industriais desde a década de 30 . São considerados universalmente como contaminantes ambientais. A importância comercial dos ftalatos é acompanhada do debate sobre seu impacto na saúde humana e ambiental. Enquanto alguns afirmam que certos ftalatos colocam em risco a saúde humana, outros grupos, geralmente associados com a produção de ftalatos, discutem que o risco posto por esses compostos é mínimo, desde que a dosagem seja em baixos níveis (Gómez-Henz e Aguilar-Caballos, 2003). Ésteres ftálicos causam distúrbios no sistema endócrino. Estudos têm mostrado que ésteres ftálicos são estrogênicos, podendo afetar o sistema reprodutor de mamíferos (Zhu, Feng e Sensenstein, 2005).

O sistema endócrino é formado pelo conjunto de glândulas que apresentam como atividade característica, a produção de secreções denominadas hormônios. Os "disruptores" endócrinos podem interferir na produção, secreção, transporte, metabolismo, efeitos de mediação e excreção de hormônios naturais, que regula o processo de desenvolvimento e suporte da homeostase endócrina no organismo (Latini et al., 2006). Devido ao potente impacto na saúde humana, a Comissão Européia propõe proibir o uso de ésteres ftálicos em PVC utilizado na fabricação de brinquedos para bebês. Nos anos 80, a Agência de Proteção Ambiental dos Estados Unidos (EPA) e muitos outros países classificaram os ésteres ftálicos como poluentes prioritários (Luks-Betlej et al., 2001). A eliminação desses compostos do meio ambiente se dá por fotólise, hidrólise química e degradação por microorganismos. A via de eliminação mais estudada é a degradação por microorganismos, já que a fotólise e a hidrólise química são reações muito lentas (Staples et al, 1997).

Estudos em animais que mostraram uma associação entre alguns ftalatos (ftalato de bis(2etil-hexila) (DEHP), ftalato de di- $n$-butila (DnBP) e ftalato de butil benzila (BBP)) e toxicidade testicular, têm gerado uma grande preocupação sobre os potenciais efeitos adversos de mudanças ambientais em consequência de lesões na saúde reprodutiva causadas por esses compostos (Fisher, 2004; Latini et al, 2006). Alguns estudos relatam que ésteres ftálicos contaminam ambientes aquáticos em locais adjacentes de indústrias, provocando toxicidade aguda ou crônica em organismos aquáticos (Chen, Chen e Chung, 2007). Na Índia, ésteres ftálicos foram encontrados em concentrações maiores em consumidores rurais e urbanos de peixes do que em vegetarianos rurais e urbanos (Rao, Pereira e Mapuskar, 2007).

A Chamada "Síndrome do Ftalato" é caracterizada por má formação do epidídimo, do ducto deferente, vesículas seminais, próstata, genitália externa, criptorquidismo (ausência do testículo na bolsa escrotal) e lesão testicular, juntamente com mudanças permanentes (feminização) na retração da aérola/mamilo (estruturas sexualmente dimórficas em roedores) e desmasculinização do crescimento do períneo, resultando na redução da distância anogenital (AGD), ou seja, a distância do ânus até a base do escroto em machos e do ânus até a base dos genitais em fêmeas (Foster, Cattley e Mychresst, 2000; Foster, 2006; Gray et al., 2007).

\section{OS QUATRO ÉSTERES DE FTALATO MAIS DEBATIDOS ATUALMENTE}

O que agrava ainda mais o quadro da utilização dos ésteres ftálicos é a baixa possibilidade real de substituí-los por outros compostos de mesma eficiência. Rahman e Brazel (2006) propuseram uma alternativa ao PVC que é a utilização de líquidos iônicos a temperatura ambiente. Os ftalatos apresentam características que um bom plastificante necessita: estabilidade, fluidez e baixa volatilidade. Entretanto, os plastificantes também devem apresentar baixa toxicidade, não ser extraível por água e alta permanência ou baixa migração, características não observadas para esses ésteres (Gómez-Henz e Aguilar-Caballos, 2003).

Então, porque os ésteres ftálicos ainda são tão utilizados na indústria? A resposta é simples. De todos os plastificantes conhecidos atualmente, os ftalatos apresentam o melhor custo benefício! Outros plastificantes que podem ser citados são: os trimetilatos (são os mais caros) e os epoxídicos que são de baixo custo, 
baixa toxicidade, mas são muito suscetíveis ao ataque de microorganismos, perdendo sua aplicabilidade em embalagens de alimentos, por exemplo (Rodolfo e Mei, 2007).

Algo que agrava a rigidez de controle do uso industrial dos ftalatos é falta de atualização dos arquivos da EPA (arquivos da IRIS Integrated Risk Information System - Sistema Integrado de Informação de Risco), desde o final da década de 80 , sobre a avaliação de risco desses compostos (Gómez-Henz e AguilarCaballos, 2003).

\section{SITUAÇÃO NO BRASIL}

O Brasil ainda não possui legislações específicas proibindo o uso de ftalatos, ao contrário de alguns países da Comunidade Européia que já proíbem os ftalatos na composição de alguns cosméticos. Nenhuma legislação brasileira limita a quantidade de ftalatos em água potável. O Conselho Nacional do Meio Ambiente - CONAMA apenas limita as concentrações de di- $n$-butil ftalato, bis(2-etilhexila) ftalto e dimetil ftalato em 0,$7 ; 1,0$ e 0,25 $\mathrm{mg} \mathrm{kg}{ }^{-1}$, respectivamente, em solos agrícolas (Conselho Nacional do Meio Ambiente CONAMA, 2006).

Algumas instituições nacionais realizam pesquisas verificando a quantidade desses compostos em diversos produtos. No ano de 2007, a empresa Faber-Castell foi notificada pela Associação de Defesa do Consumidor (Pro Teste) devido ao fato de sua borracha escolar TK Plast conter $5 \%$ em massa de ftalatos, ou seja, 50 vezes superior ao limite permitido por lei. O mais estranho desse caso, é que o produto possuía o selo do INMETRO. A Faber-Castell retirou os ftalatos do produto notificado em 13 de setembro de 2007 e incluiu nas capinhas a sigla "FC", indicando que o produto não contém ftalatos (Pro Teste, 2009).

O Instituto Brasileiro do Consumidor (Idec) realizou testes com 17 brinquedos e em oito deles, foram encontrados ftalatos. Em alguns casos havia mais de $30 \%$ da substância além do limite permitido (Instituto Brasileiro de Defesa do Consumidor, 2010).

Os quatro ésteres ftálicos mais debatidos atualmente em relação a sua larga utilização e impactos na saúde humana e meio ambiente são: ftalato de di- $n$-butila (DnBP), ftalato de butil benzila, (BBP), adipato de bis(2-etil-hexila) (DEHA) e o ftalato de bis(2-etil-hexila) (DEHP).

\section{FTALATO DE DI-n-BUTILA (DnBP)}

O DnBP é utilizado na preparação de repelentes de insetos, revestimentos para papel, solvente para tinturas e como agente de lubrificação têxtil. $\mathrm{O}$ DnBP é comumente encontrado em revestimentos de drogas em concentrações de até $9000 \mu \mathrm{g}$ por cápsula (Benson, 2009; Wormuth, 2006). A Figura 1 exibe a estrutura química do ftalato em questão.

O tempo de meia-vida do DnBP em solução aquosa é de 22 anos. É reportado na literatura (Sarma et al., 2007; Liao, Yen e Wang, 2006) que a degradação de DnBP no meio ambiente pode ocorrer facilmente por hidrólise, por fotodegradação e por biodegradação. O DnBP afeta o sistema endócrino e produz mudanças acentuadas no crescimento e desenvolvimento dos órgãos reprodutores masculinos. O DnBP age como um antiandrógeno, alterando a indicação do caminho dependente ou independente durante $\mathrm{o}$ desenvolvimento do sistema reprodutor masculino. Em ratos machos, os efeitos do DnBP no desenvolvimento do trato reprodutor são associados com a diminuição da produção de testosterona, como resultado da baixa regulação de genes envolvidos no transporte de colesterol e do caminho da síntese da testosterona. Entretanto, não são completamente entendidos os mecanismos pelos quais o DnBP mostram esses efeitos (KIM et al., 2007).

A fonte maior de exposição para o DnBP é na alimentação, possivelmente nos peixes e nos frutos do mar. Níveis em peixes variando de 78 a $200 \mathrm{ppb}$ foram quantificados. Níveis entre 3,3 e 5,7 $\mathrm{ng} \mathrm{m}^{-3}$ foram detectados no ar, próximo da cidade de Nova lorque. Em quartos recém revestidos com materiais à base de PVC, os níveis de DnBP variaram de 15000 a 26000 ng $\mathrm{m}^{-3}$ (Agency for Toxic Substances and Disease Registry (ATSDR), 1990). DnBP foi encontrado em algumas amostras de água potável nos EUA, fornecendo valores entre 0,5 e 5 ppb (U.S. Environmental Protection Agency, 2010).

Dos Santos et al. (2009) estudaram a 
presença de ftalatos em embalagens de soros fisiológicos. O DnBP foi o ftalato encontrado em maior quantidade em todas as amostras, seguido por DEHP, DEHA e BBP.

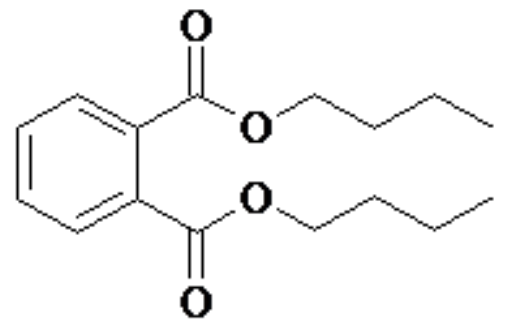

Figura 1. Estrutura química do ftalato de di-n-butila

\section{FTALATO DE BUTIL BENZILA (BBP)}

O BBP (Figura 2) é amplamente usado como plastificante de revestimentos de assoalhos à base de PVC. Também se encontra em adesivos, seladores e espumas de PVC (Mersiowsky, Weller e Ejlertsson, 2001). Esse ftalato sujeita, potencialmente, os humanos a uma exposição de $2-6 \mu \mathrm{g} \mathrm{kg}{ }^{-1} \mathrm{dia}^{-1}$. Em mamíferos, o BBP é metabolizado a ftalato de mono-benzila $(\mathrm{mBeP})$ e ftalato de mono-butila (mBuP) no sistema gastrointestinal e excretado na urina. O produto metabólico do ftalato monoéster tem toxicidades similares ao éster original (Tyl et al., 2004; IPCS, 1999). A concentração de BBP no ar dentro de lojas de materiais de construção é avaliada em $35 \mathrm{ng} \mathrm{m}^{-3}$. Há pesquisas realizadas sobre o efeito do BBP na reprodução e metabolismo de $\mathrm{Zn}$ em ratos. Também em ratos, foram realizados estudos em que se comprovou que a exposição materna ao BBP causa efeitos diversos em seus descendentes (Erma e Miyawaki, 2002; Piersma et al., 2000).

Com base na sua ampla distribuição, o BBP é considerado um dos mais importantes poluidores sintéticos do planeta e é motivo de alta preocupação para a saúde pública (UriuAdams et al., 2001; Liu e Chen, 2006). Entretanto, entre os ftalatos, o BBP é o que menos afeta o ambiente aquático. Além de possuir uma baixa solubilidade em água, o BBP tende a se repartir em sedimentos. Em ambiente aquático, o BBP tem uma rápida taxa de biodegradação (Gledhill et al., 1980).<smiles>CCCOC(=O)c1ccccc1C(=O)OCc1ccccc1</smiles>

Figura 2. Estrutura química do ftalato de butil benzila

\section{ADIPATO DE BIS(2-ETIL-HEXILA) (DEHA)}

O DEHA (Figura 3) é vulgarmente conhecido como um ftalato apesar de ser um adipato. O DEHA surgiu como um composto para substituir os ftalatos DnBp, BBP e DEHP devido aos seus efeitos. Entretanto, a sua toxicidade é conhecida por causar, entre outras coisas, proliferação de peroxisoma hepática e infertilidade em ratos (Bergman e Alabanus, 1987; Lake et al., 1997). Há poucos estudos envolvendo os danos causados pelo DEHA no sistema reprodutivo, se é que eles existem (IARC, 2000).

O DEHA é extensamente usado como plastificante. É empregado em filmes de PVC usados para embrulhar alimentos (Goulas et al., 2000). Quando esses filmes entram em contato com alimentos gordurosos, como queijo e carne, uma migração significativa de DEHA pode ocorrer para os alimentos em quantidade que podem excecer $18 \mathrm{mg}$ DEHA kg-1. Esse é o valor limite proposto pelo Comitê Europeu para Alimentos (Dalgaard et al., 2003). A quantidade de plastificante que migra da embalagem para o alimento depende de muitos fatores como o conteúdo do alimento, a concentração do plastificante na embalagem, a maneira e a temperatura em que o alimento foi estocado e também a área de contato (Petersen e Naamansen, 1998).

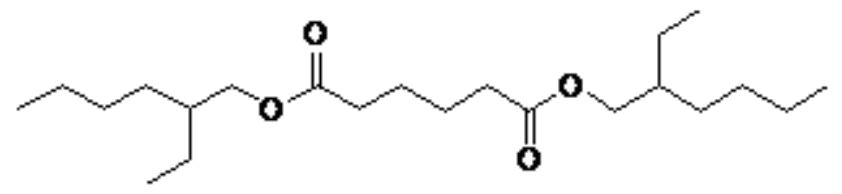

Figura 3. Estrutura química do adipato de bis(2etil-hexila) 
Kozyrod e Ziaziaris (1989) determinaram a quantidade de DEHA em vários tipos de queijo e carnes embrulhados em filmes de PVC. Valores entre 31 e $429 \mathrm{mg} \mathrm{kg}-1$ e 49 e $151 \mathrm{mg}$ $\mathrm{kg}-1$ foram encontrados, respectivamente. A Agência de Proteção Ambiental dos Estados Unidos fixou, para o DEHA, uma concentração máxima admissível em água potável em $0,4 \mathrm{mg}$ $\mathrm{L}-1$, valores acima desse pode causar um aumento do fígado e distúrbios no sistema reprodutor (Pocurrul et al, 2001; U.S. Environmental protection Agency, 2009).

\section{FTALATO DE BIS(2-ETIL-HEXILA) (DEHP)}

O DEHP (Figura 4) é o ftalato mais abundante no ambiente. Ele é o plastificante mais comumente utilizado no mundo. É liberado no meio ambiente por volatilização e lixiviação de plásticos e outras fontes ( $\mathrm{Ge}$ et al., 2007). É o principal plastificante de utensílios médicos feitos de PVC, como bolsas para sangue ou nutrição parenteral, tubulações e catéteres. Pacientes que sofrem hemodiálises e transfusões de sangue estão potencialmente sujeitos a apresentarem elevados teores de ftalatos (Wittassek, 2007). Seu uso difundido somado com a persistência no meio ambiente resulta de sua presença no ambiente e na biota (Liao, Yen e Wang, 2006). Em mamíferos, incluindo o homem, o DEHP é rapidamente hidrolisado para MEHP (Figura 5), cujo composto é mais tóxico que o próprio DEHP, e 2-hetil-hexanol. Mais tarde é oxidado a ácido 2-etil-hexanóico (Gómez-Henz e AguilarCaballos, 2003).

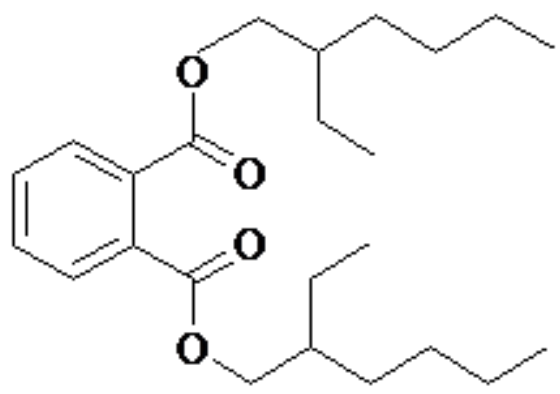

Figura 4. Estrutura química do ftalato de bis(2etil-hexila)

O DEHP também causa, como outros ftalatos, efeitos tóxicos no sistema reprodutor masculino e feminino e interfere no desenvolvimento da descendência (Kaclock et al., 2002). O DEHP provoca a síndrome metabólica, alterando o índice de massa corporal, causa obesidade abdominal e resistência à insulina (Fromme et al., 2007).

Balafas, Shaw e Whitfield (1999) investigaram a presença de seis ftalatos em 136 materiais utilizados para embalagens de alimentos na Austrália. O DEHP foi encontrado em todas as amostras.

A EPA fixou uma concentração máxima admissível em água potável em $6 \mu \mathrm{g} \mathrm{L}^{-1}$. A Agência para Substâncias Tóxicas e Registros de Doenças (ATSDR) estima a exposição máxima diária de DEHP para a população geral de $2 \mathrm{mg}$ por dia. Entretanto, a exposição ao DEHP em transfusões de sangue pode ser maior que $300 \mathrm{mg}$, equivalente a uma dose de $4,3 \mathrm{mg}$ $\mathrm{kg}^{-1}$ para um adulto pesando $70 \mathrm{~kg}$ (Ge et al., 2007).

No Brasil, a Agência Nacional de Vigilância Sanitária (ANVISA) estipula que, para o armazenamento de alimentos é permitida a presença de DEHP em até $3 \%$ da matéria plástica (Agência Nacional de Vigilância Sanitária, 2010).

\section{CONCLUSÕES:}

O limite de segurança para a exposição humana aos ésteres de ftalato ainda é motivo de muito debate entre cientistas em todo mundo. A única certeza que se tem é sua presença em diversos produtos de bens de consumo. Crianças e bebês são os mais afetados por essa exposição. Inclusive, algumas recomendações podem ser dadas para que essa exposição seja minimizada em crianças, como, por exemplo, procurar pintar ou colar carpetes no quarto com vários meses de antecedência do nascimento da criança; comprar chupetas e mordedores sem aditivos; não conservar o alimento do bebê em filmes de PVC; ao comprar algum produto para perfumar o bebê, ler o rótulo do produto para evitar a presença de ftalatos; comprar mamadeiras com o material plástico mais rígido, pois é provável que contenham uma menor quantidade desses compostos.

Diversos estudos já quantificaram ftalatos em diversas matrizes, e mesmo que ainda não exista uma conclusão definitiva sobre o tema, os valores encontrados não podem ser ignorados já 
que esses compostos são nocivos ao meio ambiente e à saúde humana.

\section{AGRADECIMENTOS:}

Ao CNPq pelo apoio financeiro.

\section{REFERÊNCIAS:}

1. Agência Nacional de Vigilância Sanitária. Boletim Alimentos e Alimentação Embalagens. Disponível em $<$ http://www.anvisa.gov.br/alimentos/alime ntacao/2005/03_05.htm>. Acesso em: 12 novembro 2010.

2. Agency for Toxic Substances and Disease Registry (ATSDR). Toxicological Profile for Di-n-Butyl Phthalate. Public Health Service, U.S. Department of Health and Human Services. 1990.

3. Balafas, D., Shaw, K.J., Whitfield, F.B. Food Chemistry, 1999, 65, 279.

4. Benson, R. Regul. Toxicol. Pharmacol. 2009, 53, 90.

5. Bergman, K., Alabanus, L. Food Chem. Toxicol. 1987, 25, 309.

6. Case Sudy: Phthalates [on-line]. Disponível em $<$ http://www.chemicalbodyburden.org/cs phthalate.htm>. Acesso em: 30 setembro 2010

7. Chen, C.Y., Chen, C.C., Chung, Y.C. Bioresour. Technol. 2007, 98, 2578.

8. Chen, M.L., Chen, J.S., Tang, C.L., Mao, I.F. Environ. Int. 2008, 34, 79.

9. Conselho Nacional do Meio Ambiente CONAMA -. Resolução $n^{\circ}$ 375, de 29 de agosto de 2006.

10. Dalgaard, M., Hass, U., Vinggaard, A.M., Jarfelt, K. Lam, H.R., Sorensen, I.K., Sommer, H.M, Ladefoged, O. Reprod. Toxicol. 2003, 17, 163.

11. Dos Santos, M.S., Martendal, E., Budiziak, D., Carasek, E. Anal. Sci. 2009, 25, 865.

12. Ema, M., Miyawaki, E. Reprod. Toxicol. 2002, 16, 71.

13. Fisher, J.S. Reproduction 2004, 127, 305.

14. Foster, P.M.D., Cattley, R.C., Mylchreest, E. Food Chem. Toxicol. 2000, 38, 597.

15. Foster, P. M. D. Int. J. Androl. 2006, 29, 140.

16. Fromme, H., Bolte, G., Koch, H.M.,
Boehmer, S., Drexler, H., Mayer, R., Liebl, B. Int. J. Hyg. Environ. Health 2007, 210, 21.

17. Ge, R.S., Chen, G.R., Tanrikut, C., Hardy, M.P. Reprod. Toxicol. 2007, 23, 366.

18. Gledhill, W.E., Kaley, R.G., Adams, W.J., Hicks, O., Michael, P.R., Saeger, V.W., Leblanc, G.A. Environ. Sci. Technol. 1980, 14, 301.

19. Gómez-Hens, A., Aguilar-Caballos, M.P. Anal. Chem. 2003, 22, 847.

20. Goulas, A.E., Anifantaki, K I., Kolioulis, D. G., Kontominas, M.G. J. Dairy Sci. 2000, 83, 1712.

21. Gray, L. E., Wilson, V. S., Howdeshell, K. L. Lambright, C. S.; Furr, J. Toxicol. Lett. 2007, 170, 177.

22. IARC. Monographs on the evaluation of carcinogenic risks to humans. Some industrial chemicals, vol. 77. Lyon: IARC Press; 2000. p. 149-75.

23. Instituto do PVC [on-line]. Disponível em:<http://www.institutodopvc.org/publico/ $?$

$\mathrm{a}=$ conteudo\&canal_id $=65 \&$ subcanal_id $=6$ 6>. Acesso em: 8 maio 2010.

24. Instituto Brasileiro de Defesa do Consumidor [on-line]. Teste com brinquedos encontra 390 vezes a quantidade de ftalatos permitida pela legislação. Disponível em $<$ http://www.idec.org.br/emacao.asp? $\mathrm{id}=1698$. . Acesso em: 09 junho 2010.

25. IPCS, Butyl benzyl phthalate, in: Concise International Chemical Assessment Document 17, World Health Organization, International Programme on Chemical Safety, Geneva, 1999.

26. Kavlock, R., Boekelheide, K., Chapin, R., Cunningham, M., Faustman, E., Foster, P., Golub, M., Henderson, R., Hinberg, I., Little, R., Seed, J., Shea, K., Tabacova, S. Tyl, R., Williams, P., Zacharewski, T. Reprod. Toxicol. 2002, 16, 529.

27. Kim, H.S., Kacew, S., Lee, B.M., Ryu, J. Y. Toxicology 2007, 234, 103.

28. Kozyrod, R.P., Ziaziaris, J. J. Food Prot. 1989, 52, 578.

29. Kummrow, F., Umbuzeiro, G.A. Quim. Nova 2006, 29, 528.

30. Lake, B. G., Brantom, P. G., Gangolli, S. D., Butterworth, K.R., Grasso, P. Toxicology 1997, 123, 217.

31. Latini, G., Del Vecchio, A., Massaro, M., 
Verotti, A., De Felice, C. Toxicology 2006, 226, 90.

32. Liao, C.S., Yen, J.H., Wang, Y.S. Chemosphere 2006, 65, 1715.

33. Liu, P.S., Chen, Y.Y., Toxicol. Appl. Pharmacol. 2006, 210, 136.

34. Luks-Betlej, K., Popp, P., Janoszka, B., Paschke, H. J. Chromatogr. A 2001, 938, 93.

35. Mackintosh, C.E., Maldonado, J.A., Ikonomu, M.G., Gobas, F.A.P.C. Environ. Sci. Technol. 2006, 40, 3481.

36. Mersiowsky, I., Weller, M., Ejlertsson, J. Water Res. 2001, 35, 3063.

37. Nogueira, R.F.P., Jardim, W.F. Quím. Nova 1998, 21, 69.

38. Petersen, J.H., Naamansen, E.T. Zeitschrift fur Lebensmittel Untersuchungund Forschung 1998, 206 , 156.

39. Piersma A.H., Verhoef, A., Biesebeek, J. D., Pieters, M.N., Slob, W. Reprod. Toxicol. 2000, 14, 417.

40. Pocurrull, E., Peñalver, A., Ferre, J., Borrul, F., Marcé, R.M.J. J. Chromatogr., A 2001, 922, 377.

41. Pro Teste [on-line]. Pro teste exige recall de borracha. Disponível em: <http://www.proteste.org.br/map/src/4599 31.htm> . Acesso em: 7 maio 2009.

42. Rahman, M., Brazel, C. Pol. Degrad. Stability 2006, 91, 3371.

43. Rao, C.V., Pereira, C., Mapuskar, K. Environ. Toxicol. Pharmacol. 2007, 23, 319.

44. Rodolfo, A., Mei, L.H.I. Polímeros: Ciên. Tecnol. 2007, 17, 263.

45. Sarma, P.N., Mohan, S.V., Shailaja, S.,
Ramakrishna, M. Bioresour. Technol. 2007, 98, 1561.

46. Staples, A.C., Peterson, D.R., Parkenton, T.H., Adams, J.W. Chemosphere 1997, 35, 667.

47. Tyl, R.W., Myers, C.B., Marr, M. C., Fail, P. A., Seely, J C., Brine, D.R., Barter, R. A., Butala, J.H. Reprod. Toxicol. 2004, 18, 241.

48. Uriu-Adams, J.Y., Kevin, R.C., Nguyen, L.K., Horvath, B.J., Nair, R., Barter, R. A., Keen, C.L. Toxicology 2001, 159, 55.

49. U.S. Environmental Protection Agency. 2009. National Primary Drinking Water Regulations: Minor Revisions to Public Notification Rule and Consumer Confidence Report Rule [on-line]. Disponível em: <http://www.epa.gov/EPAWATER/2001/S eptember/Day-07/w22522.htm>. Acesso em: 26 maio 2009.

50. U.S. Environmental Protection Agency [on-line]. Dibutyl phthalate. Disponível em $<$ http://www.epa.gov/ttn/atw/hlthef/di-nbut.html>. Acesso em: 08 julho 2010.

51. U.S. National Library of Medicine [on-line]. Pthalates. Disponível em $<$ http://toxtown.nlm.nih.gov/text_version/c hemicals. php?id=24>. Acesso em: 21 maio 2009.

52. Wittassek, M. Wismuller, G.A., Koch, H.M., Eckard, R., Dobler, L., Muller, J., Angerer, J., Schuter, C. Int. J. Hyg. Environ. Health 2007, 210, 319.

53. Wormuth, M., Risk Anal. 2006, 26, 803.

54. Zhu, J., Feng, Y.L., Sensenstein, R. Anal. Chim. Acta 2005, 538, 41.<smiles>CCCCC(CC)COC(=O)c1ccccc1C(=O)OCC(CC)CC</smiles>

DEHP
Esterase<smiles>CCCCC(CC)COC(=O)c1ccccc1C(=O)O</smiles>

MEHP

Figura 5. O metabolismo de DEHP para MEHP em animais. 